\title{
AMORTECIMENTO ATIVO DO FLUXO EM MÁQUINAS TRIFÁSICAS DE DUPLA ALIMENTAÇÃO CONTROLADAS PELAS CORRENTES ROTÓRICAS
}

\author{
Christian R. Kelber \\ e-mail: kelber@eletrica.unisinos.br \\ Universidade do Vale do Rio dos Sinos - UNISINOS \\ Centro de Ciências Exatas e Tecnológicas / Eng. Elétrica \\ Av. Unisinos 950 \\ 93022-000 São Leopoldo, RS \\ Brasil
}

Resumo - Devido à faixa limitada de ajuste da velocidade e dos baixos custos do conversor eletrônico a máquina trifásica de dupla alimentação representa uma boa opção para usinas hidrelétricas com geração de energia em velocidade ajustável e frequência fixa. Entretanto, em algumas condições de operação, o fluxo do estator deste tipo de máquina começa a apresentar oscilações. Essas oscilações são fracamente amortecidas e, caso os limites de estabilidade sejam atingidos, não poderão mais ser amortecidas sem alguma influência externa. Neste artigo serão apresentados os fundamentos do amortecimento ativo do fluxo em máquinas trifásicas de dupla alimentação através de diferentes procedimentos. Resultados experimentais, obtidos em um protótipo de laboratório com uma máquina de $33 \mathrm{~kW}$, também estão incluídos.

Abstract - Due to limited range of speed adjustment and reduced costs of the converter, doubly-fed AC machines are becoming more common in hydroelectric power plants with adjustable speed constant frequency (ASCF) generation systems. Unfortunately, under some operating conditions the stator flux of this type of machine begins to oscillate. These oscillations are poorly damped and, if the stability limits are reached, they cannot be damped without any external influence. In this paper the fundamentals of the active flux damping procedure for doubly-fed AC machines using different methods will be presented. Experimental results obtained with a $33 \mathrm{~kW}$ test rig are also included.

\section{NOMENCLATURA}

$\begin{array}{cl}\underline{\underline{X}}, \underline{x}, X & \text { Matriz, Vetor, Valor Eficaz } \\ x_{r e f} & \text { Valor de referência } \\ i_{m s} & \text { Corrente de magnetização } \\ \mu & \text { Ângulo do vetor de fluxo } \\ \omega_{m s} & \text { Velocidade angular do vetor de fluxo } \\ \omega_{o} & \text { Freqüência da rede em rad/seg } \\ i_{r d}{ }_{r d} / u^{\prime}{ }_{r d} & \text { Corrente/Tensão rotórica no eixo direto } \\ i_{r q}^{\prime} / u^{\prime}{ }_{r q} & \text { Corr./Tensão rot. no eixo em quadratura } \\ M_{e l} & \text { Torque elétrico } \\ P_{p} & \text { Par de pólos } \\ \omega_{m} & \text { Velocidade angular do rotor } \\ L_{s}, L_{r}, L_{h s} & \text { Indutâncias (estator, rotor e mútua) } \\ R_{s}, R^{\prime}{ }_{r} & \text { Resistências (estator e rotor) } \\ \sigma_{s}, \sigma_{r}^{\prime}, \sigma & \text { Fator de dispersão (estator, rotor e total) } \\ u_{n}, u_{s}, u_{r}, u_{u} & \text { Tensão (rede, estator, rotor e inversor) }\end{array}$

\author{
Walter Schumacher \\ e-mail: schumacher@ifr.ing.tu-bs.de \\ Institut für Regelungstechnik, \\ TU Braunschweig \\ Hans-Sommer-Str. 66 \\ 38106 Braunschweig \\ Alemanha
}

\section{INTRODUÇÃO}

Ao longo da vida útil de uma usina hidrelétrica que emprega turbinas hidráulicas de reação, por exemplo Francis e Kaplan, as variáveis básicas, tais como altura de queda e fluxo, podem ter seus valores alterados em relação aos valores utilizados durante o projeto da usina, obrigando, desta forma, a turbina hidráulica a operar por longos períodos de tempo fora da região de rendimento máximo, para qual ela foi projetada, implicando, assim, na redução da eficiência total da usina. Caso a velocidade da turbina possa ser alterada, esta poderá operar novamente num ponto de operação ótimo, ou seja, com rendimento máximo para as condições de operação da usina naquele instante. Além disso, a possibilidade de variação da velocidade da turbina também pode contribuir positivamente para a estabilidade do sistema elétrico [1].

O uso da máquina trifásica de dupla alimentação (MTDA) com conversor estático regenerativo em seu circuito rotórico como sistema de geração de energia com velocidade ajustável e frequência fixa (VAFF), esboçado na Figura 1, é cada vez mais comum, chegando a potências instaladas de até $400 \mathrm{MW}$ [2]. Para uma faixa pequena de variação de velocidade em torno da velocidade síncrona e com a possibilidade de geração de potência reativa, o conversor estático, ao contrário dos conversores empregados em sistemas back-to-back, possui potência nominal na ordem de $20 \%$ da potência nominal da máquina, apresentando por isso a melhor razão custo/benefício dentre as diferentes tecnologias para geração VAFF [2],[3]. O controle da máquina é realizado através da imposição das correntes rotóricas e, através destas, pode-se controlar as potências ativa e reativa do gerador de forma desacoplada e independente da velocidade da turbina [4].

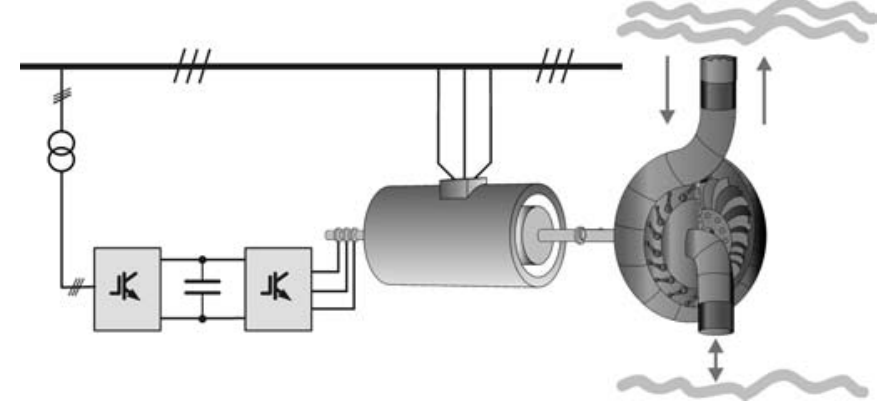

Figura 1: Usina Reversível empregando como Motor/Gerador a Máquina Trifásica de Dupla Alimentação

Construtivamente, a máquina trifásica de dupla alimentação não difere de um motor de indução com rotor 
bobinado, anéis e escovas. Devido a este fato, muitas vezes ela também é chamada de máquina de indução com dupla alimentação, quando acionada pelo estator e pelo rotor por duas fontes de tensão trifásica distintas. Entretanto, conforme [5], esta denominação apenas indica o tipo de ligação, e não o princípio de funcionamento da máquina, que nestes casos comporta-se de maneira equivalente a uma máquina síncrona, chegando inclusive a ser citada como máquina síncrona com enrolamento de campo trifásico. Da mesma forma, o termo máquina assíncrona de dupla alimentação refere-se, a princípio, aos sistemas Sherbius e Krämer estáticos, onde a máquina funciona pelos princípios físicos de um motor de indução convencional e a potência de escorregamento é enviada de volta à rede ou convertida em potência mecânica através de uma máquina secundária [6]. No presente caso, o princípio de funcionamento baseia-se na imposição das correntes rotóricas, visando-se a possibilidade do controle independente das potências ativa e reativa no lado do estator [6]. Tendo vista este fato, a máquina perde completamente as características de funcionamento como máquina síncrona ou de indução, sendo então o termo máquina trifásica de dupla alimentação mais adequado para o sistema e a estratégia de controle aqui apresentados.

Em algumas publicações são feitas investigações quanto à estabilidade da MTDA [7],[8],[9],[10], entretanto, apenas em [11] são apresentados, pela primeira vez, os limites de estabilidade em função do ponto de operação da máquina com controle vetorial baseado no fluxo do estator. No caso de distúrbios, como faltas ou mudanças rápidas nas referências das correntes rotóricas, a MTDA necessita chegar a um novo ponto de operação em um curto espaço de tempo. A resposta do sistema a esses fenômenos transitórios pode causar um deslocamento no fluxo do estator, como mostra a Figura 2, que pode ser visualizado como oscilações no módulo do vetor espacial da corrente de magnetização do estator $\left(i_{m s}\right)$.

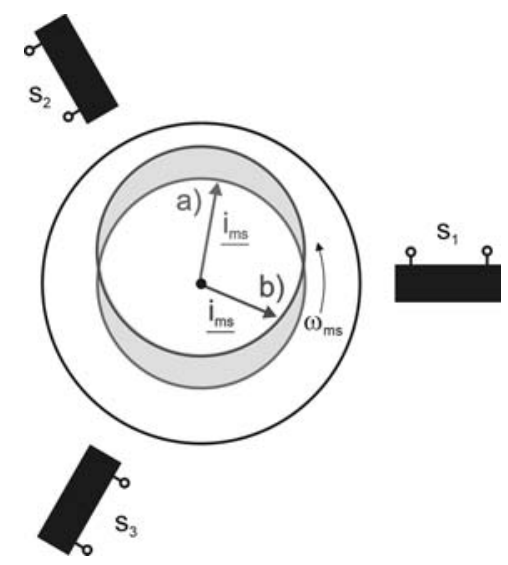

Figura 2: Deslocamento do fluxo no estator.
a) $i_{m s}$ em regime permanente.
b) $\underline{i}_{m s}$ após mudança brusca das referências.

Essas oscilações são, normalmente, fracamente amortecidas, mas em alguns casos, quando o limite de estabilidade definido em [11] é alcançado, a constante de amortecimento do sistema torna-se nula e as oscilações não mais desaparecem. Como consequência deste fato surgem oscilações no fluxo de potência entre a máquina e a rede, além de vibrações intensas, que podem comprometer, através de esforços mecânicos, a estrutura da máquina [12].

Diferentes propostas para amortecer essas oscilações são apresentadas em [13], sendo que as principais soluções serão descritas a seguir.

\section{ESTABILIDADE DA MÁQUINA TRIFÁSICA DE DUPLA ALIMENTAÇÃO}

Com base em [6] e conforme apresentado em [13], o modelo matemático da parte elétrica da MTDA pode ser apresentado na referência do fluxo do estator através das equações (1-5).

$$
\begin{aligned}
& \frac{d i_{m s}}{d t}=-\frac{u_{n}}{L_{h s}} \operatorname{sen}\left(\omega_{o} t-\mu\right)+\frac{R_{s}}{L_{s}} i_{r d}^{\prime}-\frac{R_{s}}{L_{s}} i_{m s} \\
& \frac{d \mu}{d t}=\omega_{m s}=\frac{u_{n}}{L_{h s} i_{m s}} \cos \left(\omega_{o} t-\mu\right)+\frac{R_{s}}{L_{s} i_{m s}} i_{r q}^{\prime} \\
& M_{e l}=-\frac{3 L_{h s} p_{p}}{\left(1+\sigma_{s}\right)} \cdot i_{r q}^{\prime} \cdot i_{m s} \\
& \frac{d i_{r d}^{\prime}}{d t}=\frac{1}{L_{r}^{\prime} \sigma} \cdot u_{r d}^{\prime}+\left(\omega_{m s}-\omega_{m} p_{p}\right) \cdot i_{r q}^{\prime}- \\
& \frac{d i_{r q}^{\prime}}{d t}=\frac{1}{L_{r}^{\prime} \sigma} \cdot u_{r q}^{\prime}-\left(\omega_{m s}-\omega_{m} p_{p}\right) \cdot i_{r d}^{\prime}- \\
& \quad-\frac{(1-\sigma)}{\sigma} \cdot\left(\omega_{m s}-\omega_{m} p_{p}\right) \cdot i_{m s}-\frac{R_{r s}^{\prime}}{L_{r}^{\prime} \sigma} \cdot i_{r q}^{\prime}{ }_{r q}^{\prime} \sigma \\
& L_{r d}^{\prime}
\end{aligned}
$$

O controle da MTDA parte do princípio da imposição das correntes rotóricas $\left(i_{r d}^{\prime}\right.$ e $\left.i_{r q}^{\prime}\right)$ [4], [6], [13], garantida pelos controladores apresentados na Figura 3.

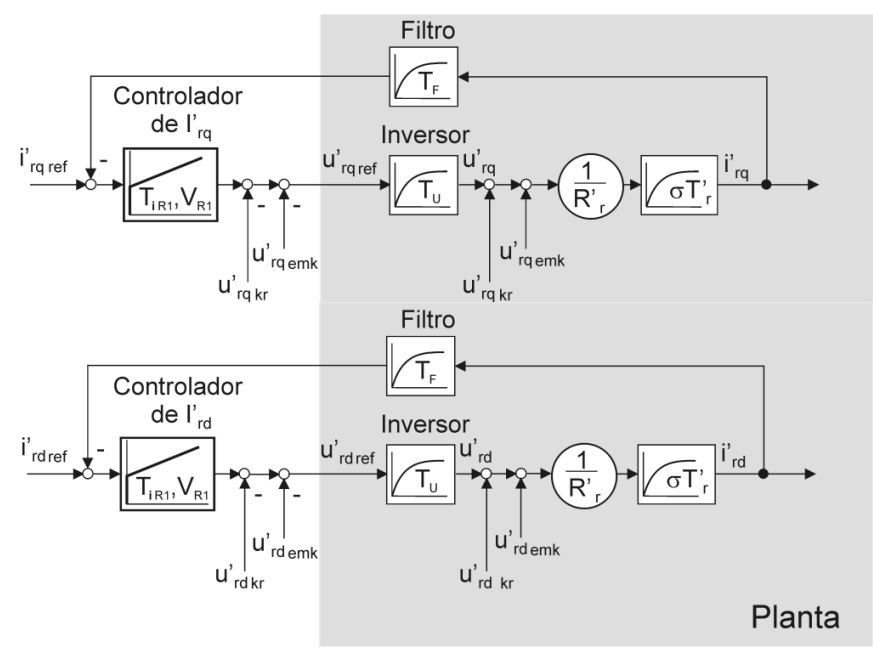

Figura 3: Controle das correntes rotóricas

Os controladores são projetados de forma a garantir um rápido seguimento da referência. Visando não sobrecarregálos, todos os termos de acoplamento cruzado entre $i_{r d}^{{ }_{r d}}$ e $i_{r q}{ }_{r q}$ $\left(u_{r d k r}^{\prime}\right.$ e $\left.u_{r q k r}^{\prime}\right)$, bem como a influência das tensões induzidas 
( $u_{r \text { r emk }}^{\prime}$ e $u_{r q \text { emk }}^{\prime}$ ), sendo considerados distúrbios conhecidos para as malhas de controle, podem ser compensados a partir de seus valores estimados. Estes valores podem ser obtidos das equações (4) e (5), onde os termos de acoplamento cruzado e as tensões induzidas estão relacionados, respectivamente, aos segundos e terceiros elementos nas igualdades.

Uma vez garantida a imposição das correntes rotóricas, o modelo da MTDA pode ser resumido às equações (1-3). A partir destas equações é possível chegar-se ao modelo linearizado da máquina para pequenas perturbações, que pode ser expressado no espaço-de-estados conforme [11]. A estabilidade da MTDA é então investigada através de simulação numérica, computando-se os autovalores do sistemas e tendo as correntes rotóricas nos eixos direto $\left(i_{r d}{ }_{r d}\right)$ e em quadratura $\left(i_{r q}{ }_{r q}\right)$ como variáveis. Os autovalores obtidos são apresentados na Figura 4 e representam um sistema fracamente amortecido com frequência natural próxima da frequência da rede $(50 \mathrm{~Hz})$.

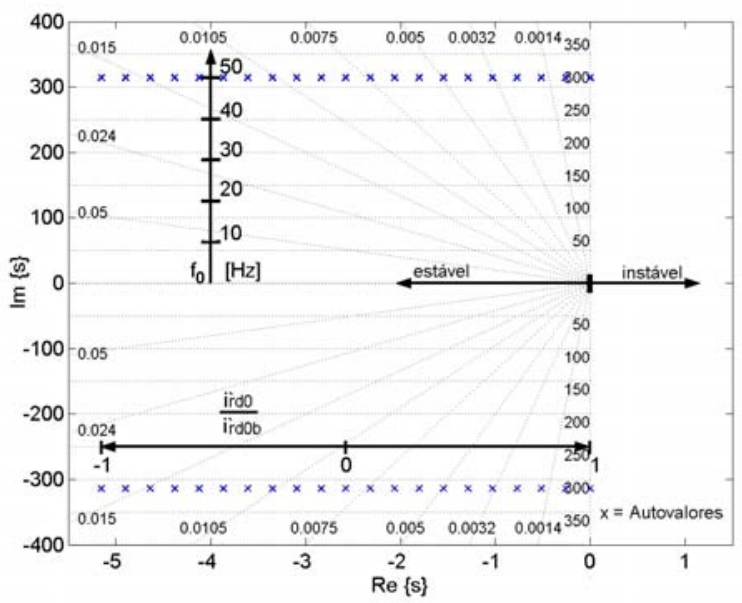

Figura 4: Autovalores para diferentes pontos de operação

Enquanto que variações na componente em quadratura da corrente rotórica deslocam os autovalores na direção paralela ao eixo imaginário, o aumento da componente direta deslocaos paralelamente ao eixo real no sentido da instabilidade. Quando $i_{r d}^{\prime}$ alcança o valor de $i_{r d 0 b}^{\prime}$, definido em [11] como a corrente no eixo direto necessária para que a máquina gere a mesma quantidade de reativo que estaria consumindo caso estivesse conectada à rede como um motor de indução convencional, os autovalores cruzam o eixo imaginário e o sistema torna-se instável.

De forma a auxiliar a análise do fenômeno, a Figura 5 apresenta as respostas de $i_{m s}$ para diferentes saltos nas componentes $d$ e $q$ das correntes rotóricas, obtidos a partir de simulação numérica com o modelo linearizado da máquina para pequenas perturbações em torno de um ponto de operação. Os dados da máquina, necessários para as simulações, encontram-se no apêndice.

Pode-se notar, nos resultados apresentados na Figura 5, que não há qualquer alteração na constante de amortecimento do sistema no caso de excitações através de $i_{r q}{ }_{r q}$, ao contrário das excitações através de $i^{\prime}{ }_{r d}$. O pior caso, visto na Figura 5.a., ocorre para o salto positivo com amplitude $i_{r d 0 b}$ aplicado em $i_{r d}^{\prime}$, quando os pólos do sistema são levados em direção à instabilidade, conforme apresentado na Figura 4.
Ao mesmo tempo pode-se verificar, que há o surgimento de uma pequena variação no valor estacionário da corrente de magnetização para uma atuação no sistema a partir de $i_{r q}^{\prime}$, enquanto que atuações a partir de $i_{r d}^{\prime}$ não influenciam no valor estacionário de $i_{m s}$. Este fato já era esperado, uma vez que, para o sistema em regime estacionário, num determinado ponto de operação, a equação (2) não apresenta qualquer relação entre $i_{r d}^{\prime}$ e $i_{m s}$.
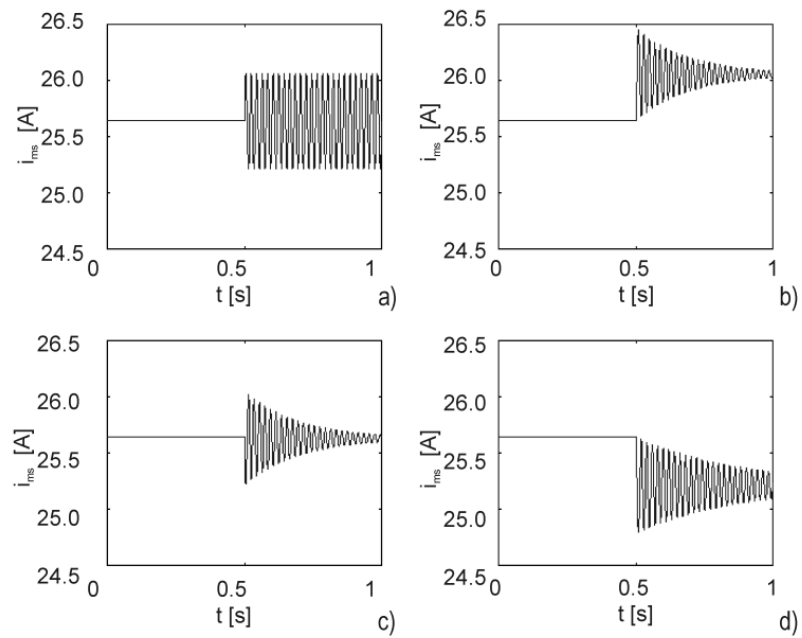

Figura 5: Resposta de $i_{m s}$ aos saltos:

$$
\begin{aligned}
& \text { a) } i_{r q}{ }_{r q}=0 \quad ; \quad i_{r d}^{\prime}: 0 \rightarrow i_{r d 0 b}{ }^{\prime} \\
& \text { b) } i_{r d}{ }_{r d}=0 \quad ; \quad i_{r q}{ }_{r q}: 0 \rightarrow i^{\prime}{ }_{r d o b} \\
& \text { c) } i_{r q}{ }_{r q}=0 \quad ; \quad i_{r d}{ }_{r d}: 0 \rightarrow-i{ }_{r d 0 b} \\
& \text { d) } i_{r d}{ }_{r d}=0 \quad ; \quad i_{r q}{ }_{r q}: 0 \rightarrow-i^{\prime}{ }_{r d 0 b}
\end{aligned}
$$

Em regime permanente, o valor de $i_{m s}$, obtido a partir de (2) e aplicado em (1), fornecerá o valor do ângulo $\alpha$, apresentado na equação (6). Este ângulo representa a diferença angular entre dois sistemas de coordenadas, o primeiro baseados no vetor da tensão da rede e o segundo no vetor da corrente de magnetização.

$$
\alpha=\omega_{o} t-\mu
$$

Em condições normais de operação, o ângulo $\alpha$ apresenta valores estacionários muito pequenos, de forma que pode ser assumida uma coincidência na direção da componente em quadratura do vetor da corrente rotórica $i_{r q}{ }_{r q}$ com a direção do vetor da tensão da rede $\underline{u_{n}}$ [4],[6],[13]. Este fato permite, portanto, uma atuação independente sobre as potências reativa e ativa no lado do estator a partir das componentes direta e em quadratura da corrente rotórica, conforme as equações (7) e (8).

$$
\begin{aligned}
& P_{s}=\frac{2}{3} \operatorname{Re}\left\{\underline{u_{s}} \cdot \underline{i_{s}}\right\} \approx-3 \cdot \frac{U_{s}}{\left(1+\sigma_{s}\right)} \cdot i_{r q}^{\prime} \\
& \left.Q_{s}=\frac{2}{3} \operatorname{Im} \underline{\underline{u_{s}}} \cdot \underline{i_{s}}\right\} \approx 3 \cdot \frac{U_{s}}{\left(1+\sigma_{s}\right)} \cdot\left(\frac{U_{s}}{L_{h s} \cdot \omega_{m s}}-i_{r d}^{\prime}\right)
\end{aligned}
$$

Chega-se, desta forma, à principal vantagem da utilização da MTDA como gerador, que está na possibilidade do controle das potências ativa e reativa de forma independente, não importando o valor da velocidade rotórica, uma vez 
mantida esta dentro da região de operação. A estrutura das malhas de controle das potências é apresentada na Figura 6, onde os controladores das potências ativa e reativa são implementados em cascata com as malhas de controle das componentes $i_{r q}^{\prime}$ e $i_{r d}^{\prime}$, respectivamente.

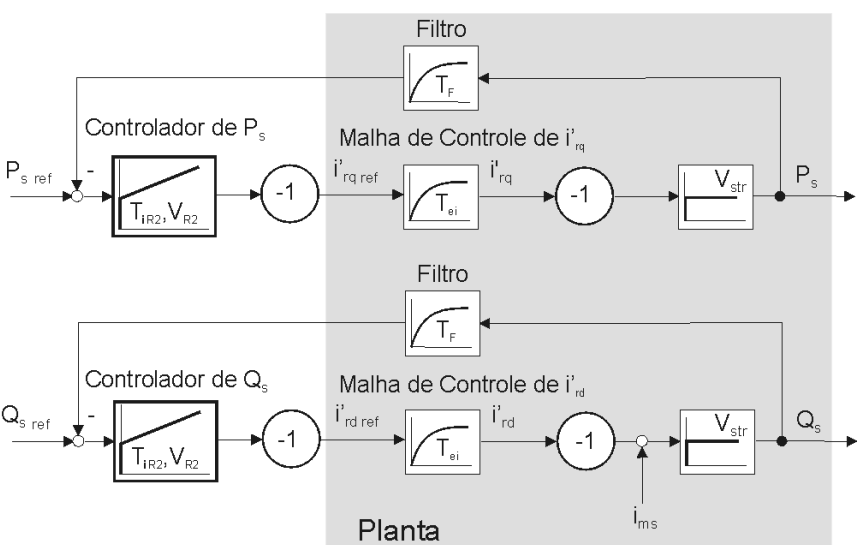

Figura 6: Controle independente das potências ativa e reativa

Independente da atuação destes controladores, as oscilações fracamente amortecidas em $i_{m s}$, apresentadas na Figura 5, provocam, por sua vez, oscilações em $\mu$, de forma que, através de (6), surgem, também, oscilações em $\alpha$, o que implica, consequentemente, em oscilações no fluxo de potências entre a máquina e a rede, mesmo garantidos valores constantes para $i_{r d}^{\prime}$ e $i_{r q}^{\prime}$. Estas oscilações também não são compensadas pelos controladores de potência, uma vez que estes encontram-se nas malhas externas de controle e são, portanto, de atuação mais lenta, não conseguindo, por sua vez, atuar sobre a causa do problema dentro das malhas internas.

\section{PROPOSTAS DE AMORTECIMENTO ATIVO}

Dentre as propostas apresentadas em [13] para amortecer as oscilações de $i_{m s}$ destacam-se três técnicas com estratégias de atuação distintas. A primeira caracteriza-se como uma solução preventiva e visa adequar os parâmetros dos controladores de corrente de forma a evitar que as oscilações possam surgir. A segunda técnica baseia-se no valor estimado de $i_{m s}$ e utiliza este sinal em uma malha de controle extra, visando, desta forma, o amortecimento ativo das oscilações. A terceira técnica, que envolve a utilização de um conversor extra situado eletricamente dentro do circuito do estator no lugar de seu ponto de neutro, visa alterar dinamicamente as características da planta de forma invisível aos controladores preexistentes.

\section{A. Amortecimento através de controladores de corrente lentos}

Esta primeira proposta de amortecimento das oscilações no fluxo do estator, apresentada primeiramente em [11], não se caracteriza efetivamente como uma técnica de amortecimento ativo, mas sim como um procedimento para evitar que as oscilações surjam. Neste caso, os parâmetros dos controladores de corrente são projetados de tal forma, que o módulo da função de transferência do sistema em malha fechada para a frequência das oscilações seja reduzido para $10 \%$ do valor nominal [13]. Desta forma, os controladores de corrente responderão de forma mais lenta, evitando, assim, variações bruscas em $i_{r d}^{\prime} \mathrm{e}$, consequentemente, o surgimento de oscilações em $i_{m s}$.

\section{B. Amortecimento baseado no valor estimado de $i_{m s}$}

Através do próprio modelo matemático da MTDA utilizado para o controle vetorial da mesma, pode-se obter o valor estimado de $i_{m s}$ ao longo do tempo. Este sinal, contendo a informação das oscilações que deverão ser amortecidas, poderia ser então utilizado de forma a compor um sinal de compensação a ser inserido nas componentes direta e em quadratura da corrente rotórica. Devido a este princípio de funcionamento este método pode ser chamado de back-ims.

De forma a verificar esta proposta foi adicionada às componentes da corrente rotórica do modelo linearizado da MTDA, apresentado em [11] e cujos vetores de estado, entrada e saída são descritos em (9), uma realimentação da primeira derivada da variável de estado $\left(i_{m s}\right)$.

$$
\underline{\Delta x}=\left[\begin{array}{c}
\Delta i_{m s} \\
\Delta \mu
\end{array}\right] ; \underline{\Delta u}=\left[\begin{array}{c}
\Delta i^{\prime}{ }_{r d} \\
\Delta i^{\prime}{ }_{r q} \\
\Delta u_{s}
\end{array}\right] ; \underline{\Delta y}=\left[\begin{array}{c}
\Delta i_{m s} \\
\Delta M_{e l}
\end{array}\right]
$$

Diferente da forma tradicional, onde é inserida no sistema uma matriz de realimentação do vetor de estados, neste caso, a sua derivada será realimentada através da matriz $\underline{\underline{K}}$, definida em (10), sendo que $k_{p}$ representa o valor de uma constante. $\mathrm{O}$ princípio deste procedimento é apresentado na Figura 7.

$$
\underline{\underline{K}}=\left[\begin{array}{cc}
-k_{p} & 0 \\
-k_{p} & 0 \\
0 & 0
\end{array}\right]
$$

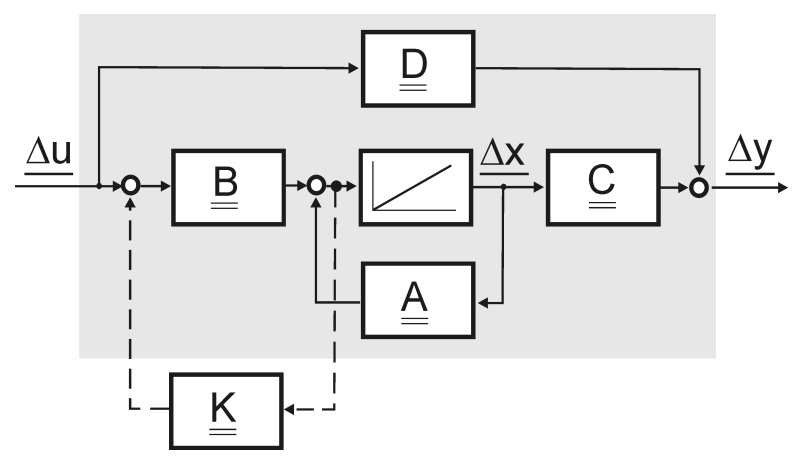

Figura 7: Proposta de controle para amortecimento back-ims

Após alguns cálculos, surge então um novo sistema, descrito no espaço-de-estados pelas matrizes $\underline{\underline{A^{\prime}}}, \underline{\underline{B^{\prime}}}, \underline{\underline{C^{\prime}}}$ e $\underline{\underline{D^{\prime}}}$, apresentadas em (11). Os vetores de estado, entrada e saída, descritos em (9), permanecem, neste caso, inalterados.

$$
\begin{aligned}
& \underline{\underline{A}}^{\prime}=(\underline{\underline{I}}-\underline{\underline{B}} \underline{\underline{K}})^{-1} \underline{\underline{A}} \\
& \underline{\underline{B}}^{\prime}=(\underline{\underline{I}}-\underline{\underline{B}} \underline{\underline{K}})^{-1} \underline{\underline{B}} \\
& \left.\underline{\underline{C}}^{\prime}=\underline{\underline{C}}+\underline{\underline{D}} \underline{\underline{K}} \underline{\underline{I}}-\underline{\underline{B}} \underline{\underline{K}}\right)^{-1} \underline{\underline{A}} \\
& \underline{\underline{D}} \cdot=\underline{\underline{D}} \underline{\underline{K}}(\underline{\underline{I}}-\underline{\underline{B}} \underline{\underline{K}})^{-1} \underline{\underline{B}}+\underline{\underline{D}}
\end{aligned}
$$


A análise da estabilidade do novo sistema, composto pela MTDA amortecida ativamente através das correntes rotóricas, é realizada a partir de simulação numérica com a obtenção dos autovalores da nova matriz sistêmica para diferentes valores de $i_{r d}{ }_{r d}$ e $i_{r q}$. Conforme é exemplificado na Figura 8, a partir de pequenos valores da constante $k_{p}$ podese obter um aumento significativo na constante de amortecimento do sistema. Para valores maiores de $k_{p}$, este aumento na constante de amortecimento é aliado à redução no valor da frequência natural.

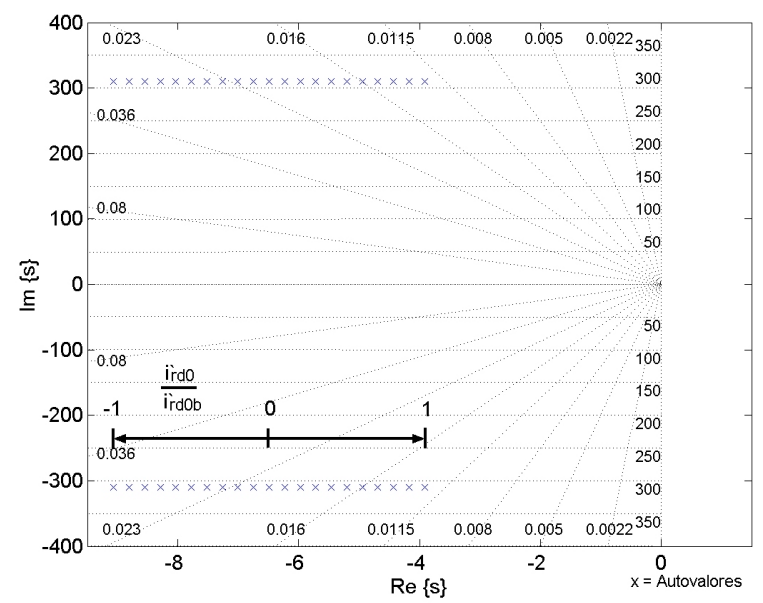

Figura 8: Deslocamento dos autovalores da MTDA através do amortecimento ativo do tipo back-ims $\left(k_{p}=0.01\right)$

\section{Amortecimento através de variação dinâmica da planta}

Para amortecer ativamente o fluxo da MTDA esta terceira proposta necessita da inclusão no sistema de um inversor adicional, conectado em série com o estator da máquina, no lugar de seu ponto neutro [13], como é mostrado na Figura 9. Um conversor com potência nominal igual a da MTDA também poderia ser utilizado para esta finalidade, sendo conectado entre a rede e a máquina, em série com seu estator numa configuração back-to-back [14],[15],[16]. Entretanto, devido ao baixo valor da tensão necessária para amortecer o fluxo do estator da máquina, este inversor adicional também pode ser dimensionado para uma porção da potência nominal da MTDA, mantendo assim o aspecto de baixo custo para sistemas de geração VAFF empregando máquinas de dupla alimentação [17].

Nesta configuração, o terceiro inversor insere um vetor espacial de tensão em série com o vetor da tensão da rede, mantendo-se nulo durante a operação da máquina livre de distúrbios. No caso da ocorrência de qualquer distúrbio é realizada, através deste inversor, uma variação dinâmica da estrutura do sistema, aumentando, desta forma, o fator de amortecimento, sem afetar os controladores de corrente no lado do rotor. Ao contrário da proposta anterior, esta configuração não gera correntes elevadas no circuito rotórico, especialmente após faltas na rede, além de chegar a um ponto de operação estável no menor intervalo de tempo dentre todas as formas de amortecimento ativo do fluxo da máquina trifásica de dupla alimentação [13],[17].

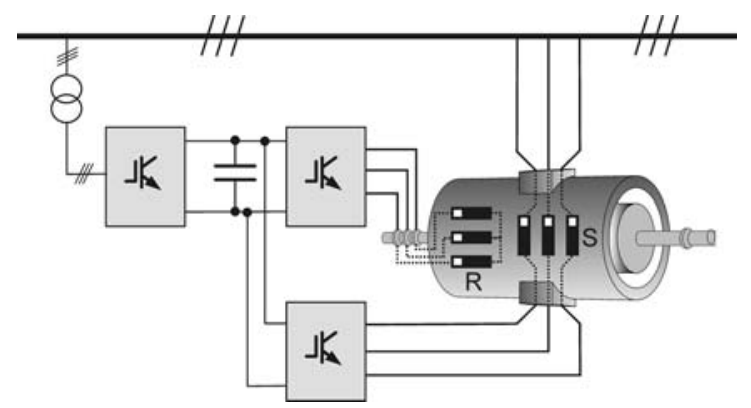

Figura 9: MTDA com conversor estendido

O vetor de tensão no estator da máquina passa a ser composto a partir dos vetores das tensões da rede e do inversor. Além disso, com a inserção do terceiro inversor, foi obtido um grau de liberdade extra no sistema, que pode ser associado à componente direta da tensão do inversor $\left(u_{u d}\right)$, sendo esta utilizada como variável de atuação sobre o circuito do estator e sem grandes interferências nas malhas dos controladores previamente existentes [13],[17].

Para definir a estrutura de controle que vai reger esta "tensão de amortecimento", algumas condições de contorno devem ser estabelecidas:

- O sistema amortecedor deve possuir uma resposta extremamente rápida.

- Os controladores das correntes no lado do rotor não devem ser afetados.

- Após o amortecimento do fluxo, a tensão do inversor deve decair à zero para o retorno das condições normais de operação, caso contrário ela afetaria a capacidade de geração independente das potências ativa e reativa por parte da MTDA controlada pelas correntes rotóricas.

Estas condições podem ser obtidas a partir de uma função de transferência de segunda ordem, dada pela equação (12). A entrada do sistema de amortecimento é, novamente, a derivada de $i_{m s}$, sinal que melhor representa as oscilações no fluxo.

$$
H(s)=\frac{\Delta u_{u d}}{\Delta \dot{i}_{m s}}=\frac{b_{2} s^{2}+b_{1} s+b_{0}}{a_{2} s^{2}+a_{1} s+a_{0}}
$$

Conforme apresentado em [17], com a introdução de um sistema amortecedor de segunda ordem ocorre a duplicação do número de variáveis de estado, sendo estas novas definidas por $d_{1}$ e $d_{2}$, bem como a introdução da tensão de amortecimento no vetor de entrada do sistema. $\mathrm{O}$ vetor de saída permanece inalterado. Os vetores de estado, entrada e saída para o novo sistema, já linearizado, são apresentados em (13).

$$
\underline{\Delta x}=\left[\begin{array}{c}
\Delta i_{m s} \\
\Delta \mu \\
d_{1} \\
d_{2}
\end{array}\right] ; \underline{\Delta u}=\left[\begin{array}{c}
\Delta i^{\prime}{ }_{r d} \\
\Delta i^{\prime}{ }_{r q} \\
\Delta u_{u d} \\
\Delta u_{s}
\end{array}\right] ; \underline{\Delta y}=\left[\begin{array}{c}
\Delta i_{m s} \\
\Delta M_{e l}
\end{array}\right]
$$

Similarmente ao procedimento apresentado em [11], para a análise do comportamento dinâmico desta nova configuração um modelo linearizado para pequenas perturbações incluindo a estrutura de amortecimento pode ser obtido e descrito no espaço-de-estados pelas matrizes $\underline{\underline{A^{\prime}}}$, 


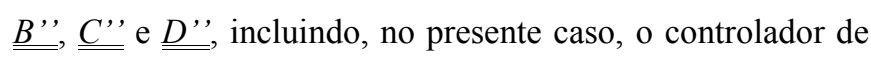
estados representado pela matriz $\underline{\underline{F^{\prime}}}$ [17], como mostra a Figura 10.

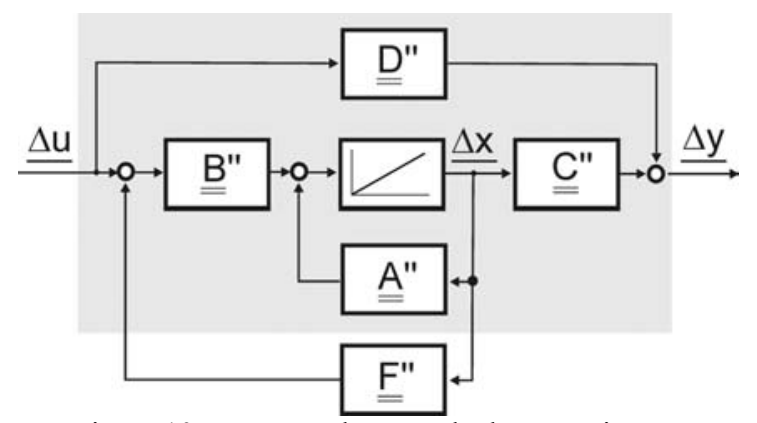

Figura 10: Estrutura de controle do novo sistema

A estabilidade da MTDA com amortecimento ativo através da variação dinâmica da planta pode ser investigada através de simulação numérica computando-se os autovalores da matriz sistêmica da malha fechada $\left(\underline{\underline{A^{\prime \prime}}}+\underline{\underline{B}} \underline{\underline{F^{\prime \prime}}}\right)$. A Figura 11 apresenta, por exemplo, o deslocamento que pode ser realizado com os pólos que originalmente encontravamse sobre o eixo imaginário.

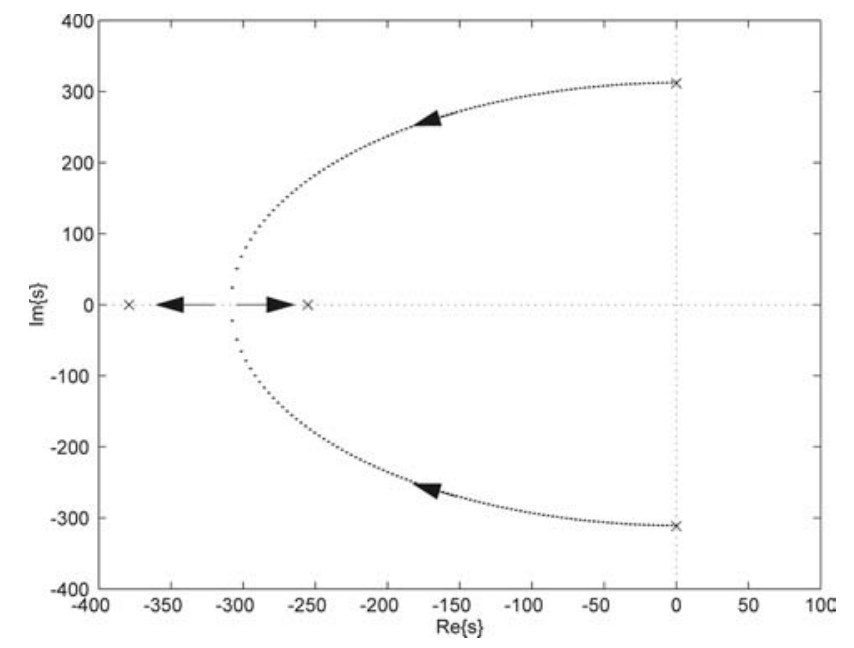

Figura 11: Deslocamento dos autovalores da MTDA através de variação dinâmica da planta.

Os parâmetros da função de transferência são definidos de forma a garantir que os autovalores permaneçam numa região estável, de forma que, quando a corrente $i_{r d}^{\prime}$ atingir o valor $i_{r d 0 b}$, os pólos, inicialmente formando um par complexo conjugado sobre o eixo imaginário do plano complexo, sejam deslocados para a esquerda até atingirem o eixo real [17].

\section{RESULTADOS EXPERIMENTAIS}

O protótipo utilizado para a realização dos ensaios consiste em uma máquina trifásica de dupla alimentação de $33 \mathrm{~kW}$, acionada por um motor de corrente contínua de $40 \mathrm{~kW}$, capaz de simular o comportamento de uma turbina hidráulica. Os dados do gerador são apresentados no apêndice.

$O$ rotor é alimentado a partir de um inversor VSI-PWM a base de IGBTs, enquanto que o estator por um lado é conectado à rede e por outro ao inversor adicional, também do tipo VSI-PWM a base de IGBTs. Ambos inversores são alimentados pelo mesmo elo de tensão DC e possuem frequência de chaveamento ajustável para $4 \mathrm{kHz}, 2 \mathrm{kHz}, 1 \mathrm{kHz}$ e $500 \mathrm{~Hz}$. O controle das correntes rotóricas, das potências ativa e reativa, bem como as estruturas de amortecimento são implementados em processadores digitais de sinais VeCon [18].

Para comprovar o surgimento de oscilações em $i_{m s}$, foi aplicado, no protótipo de laboratório, um degrau de amplitude $i_{r d 0 b}^{\prime}$ na referência da componente direta da corrente rotórica $\left(i^{\prime}{ }_{r d r e f}\right)$. A Figura 12 apresenta o resultado para os conversores operando a uma frequência de chaveamento de $4 \mathrm{kHz}$, enquanto que na Figura 13 o mesmo ensaio é realizado para uma frequência de chaveamento de $2 \mathrm{kHz}$.

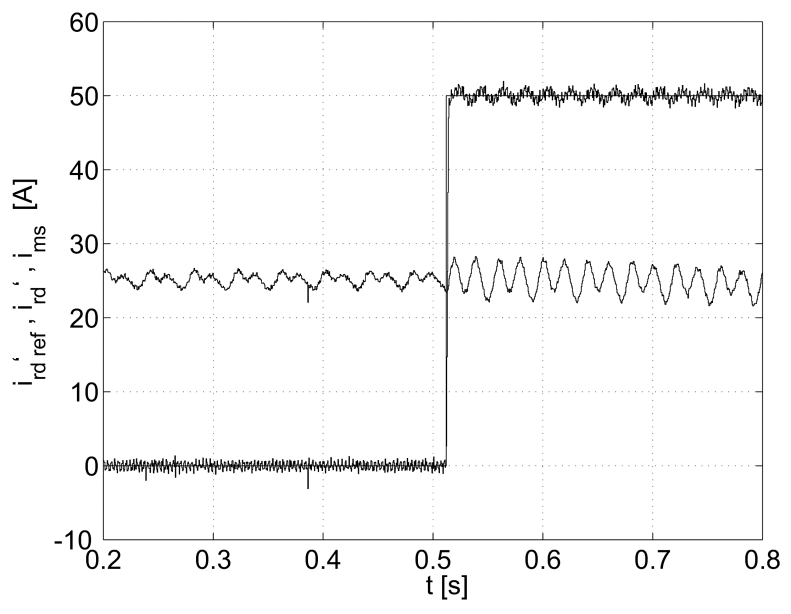

Figura 12: Resposta de $i_{m s}$ ao salto em $i_{r d ~ r e f}^{\prime}(\mathrm{PWM}=4 \mathrm{kHz})$

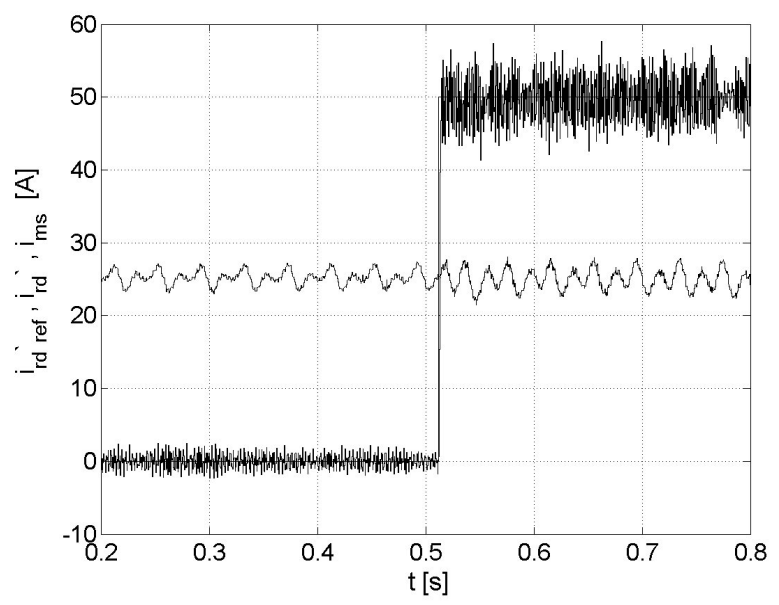

Figura 13: Resposta de $i_{m s}$ ao salto em $i_{r d ~ r e f}(\mathrm{PWM}=2 \mathrm{kHz})$

Pode-se verificar que, após o salto, $i_{m s}$ apresenta uma oscilação não amortecida com frequência próxima à frequência da rede, independentemente da frequência de chaveamento do inversor.

Após estes ensaios, os parâmetros dos controladores das correntes $i_{r d}^{\prime}$ e $i_{r q}^{\prime}$ foram reprojetados e ajustados de tal forma que estes, reagindo lentamente, atenuassem a resposta do sistema em malha fechada para frequências próximas à frequência da rede. O resultado deste procedimento é apresentado na Figura 14. Pode-se perceber uma resposta ao 
salto lenta por parte da componente no eixo direto da corrente rotórica, que passa a apresentar um pequeno sobressalto. Conforme era esperado, não houve, neste caso, o surgimento em $i_{m s}$ de oscilações com a frequência da rede.

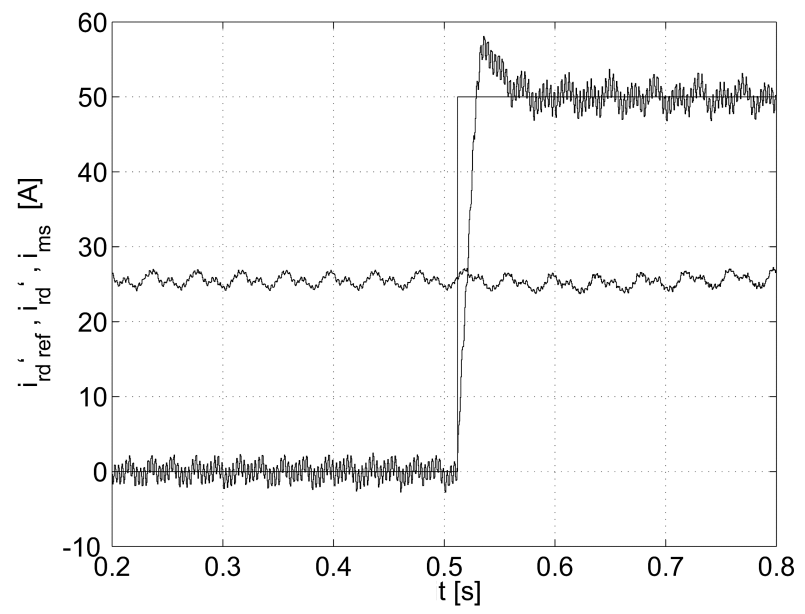

Figura 14: Resposta de $i_{m s}$ ao salto em $i_{r d}{ }_{r e f}$ para o sistema com controladores de corrente lentos $(\mathrm{PWM}=4 \mathrm{kHz})$

O amortecimento através da utilização de controladores de corrente lentos apresenta uma boa qualidade, sem quaisquer custos adicionais em hardware ou software. Entretanto, mesmo com este procedimento, os pólos da máquina, estando ela gerando potência reativa, continuam próximos ao eixo imaginário, de forma que outros distúrbios, como faltas, podem vir a causar o surgimento de oscilações fracamente amortecidas no fluxo.

$\mathrm{Na}$ Figura 15 é apresentada a resposta de $i_{m s}$ para um salto

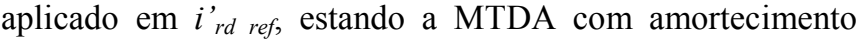
ativo back-ims e utilizando os mesmos controladores de corrente empregados no ensaio para a obtenção da resposta do sistema original, apresentada na Figura 12. Comparandose os dois resultados, percebe-se que, utilizando-se a proposta de amortecimento ativo do tipo back-ims, mesmo com a utilização de controladores de corrente rápidos, as oscilações em $i_{m s}$ com frequência da rede são fortemente amortecidas.

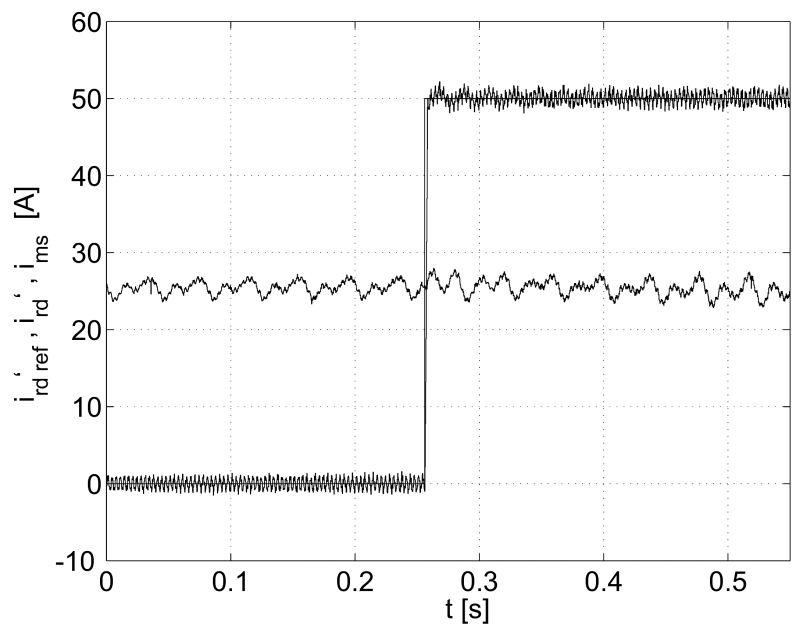

Figura 15: Resposta de $i_{m s}$ ao salto em $i_{r d}^{\prime}$ ref para o sistema com amortecimento ativo do tipo back-ims $(\mathrm{PWM}=4 \mathrm{kHz})$
A proposta de amortecimento ativo back-ims, ou seja, baseado na realimentação da primeira derivada do valor estimado de $i_{m s}$, apresenta uma excelente qualidade no amortecimento do fluxo. Com nenhum custo adicional em hardware, necessita apenas da inclusão, no software de controle, das rotinas responsáveis pelo amortecimeto ativo. Apresentando similaridades com a solução proposta em [19], este procedimento possui, da mesma forma, problemas com correntes rotóricas elevadas, que podem surgir após faltas no sistema elétrico, causando, consequentemente, surtos transitórios nas potências ativa e reativa no lado do estator da máquina. Devido à característica transitória destes fenômenos, estes até podem ser desprezados dentro de certos limites, desde que haja uma adequação dinâmica do parâmetro $k_{p}$ [13].

Na Figura 16 é apresentada a resposta de $i_{m s}$ para um salto aplicado em $i_{r d}^{\prime}$ ref, estando a máquina operando com amortecimento ativo através de variação dinâmica da planta. Neste ensaio os inversores tiveram a frequência de chaveamento reduzida de $4 \mathrm{kHz}$ para $2 \mathrm{kHz}$. Comparando este resultado com a resposta da máquina não amortecida, apresentada na Figura 13, percebe-se também, neste caso, o desaparecimento das oscilações em $i_{m s}$ com frequência de $50 \mathrm{~Hz}$.

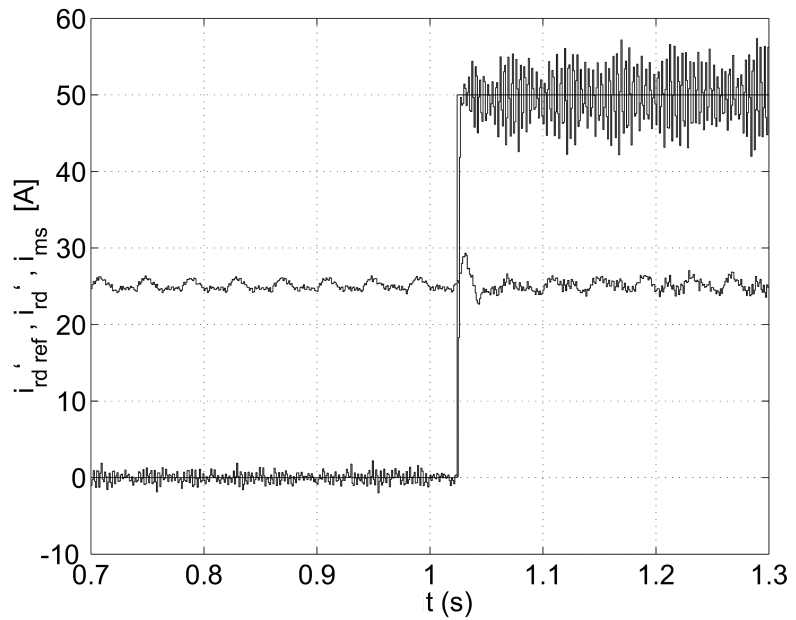

Figura 16: Resposta de $i_{m s}$ ao salto em $i_{r d \text { ref }}^{\prime}$ para o sistema com variação dinâmica da planta $(\mathrm{PWM}=2 \mathrm{kHz})$

A técnica de amortecimento ativo do fluxo através de variação dinâmica da planta do sistema apresenta excelentes resultados, possibilitando uma estabilização da máquina de forma extremamente rápida. Entretanto, a necessidade de um inversor extra, aliado a toda infra-estrutura necessária para sua proteção, torna o sistema extremamente caro, a nível de software e, principalmente, a nível de hardware. No caso de mudanças bruscas no sistema de potência, como faltas ou rejeição de carga, a MTDA amortecida ativamente através deste método alcança um ponto de operação estável em poucos milésimos de segundo, similarmente ao resultado apresentado na Figura 16, podendo, desta forma, contribuir positivamente para a estabilidade do sistema elétrico.

A Tabela 1 apresenta, de forma resumida, um quadro comparativo entre as diferentes estratégias de amortecimento das oscilações em $i_{m s}$. A escolha da estratégia a ser implementada, entretanto, dependerá das necessidades exigidas à MTDA. 
Tabela 1: Quadro comparativo das propostas de amortecimento ativo apresentadas

\begin{tabular}{c|ccc}
\hline & $\begin{array}{c}\text { Amortecimento } \\
\text { através de } \\
\text { controladores de } \\
\text { corrente lentos }\end{array}$ & $\begin{array}{c}\text { Amortecimento } \\
\text { através da } \\
\text { técnica back-ims }\end{array}$ & $\begin{array}{c}\text { Amortecimento } \\
\text { através de } \\
\text { variação dinâmica } \\
\text { da planta }\end{array}$ \\
\hline $\begin{array}{c}\text { Qualidade do } \\
\text { amortecimento }\end{array}$ & Boa & Excelente & Excelente \\
\hline $\begin{array}{c}\text { Custo em } \\
\text { hardware } \\
\text { adicional }\end{array}$ & Nenhum & Nenhum & $\begin{array}{c}\text { Extremamente } \\
\text { Elevado }\end{array}$ \\
\hline $\begin{array}{c}\text { Custo em } \\
\text { software } \\
\text { adicional }\end{array}$ & Nenhum & Baixo & Elevado \\
\hline $\begin{array}{c}\text { Sobressalto de } \\
\text { corrente } \\
\text { (após faltas) }\end{array}$ & Baixo & Extremamente & Elevado \\
\hline $\begin{array}{c}\text { Estabilização } \\
\text { após faltas }\end{array}$ & Muito lenta & Rápida & $\begin{array}{c}\text { Extremamente } \\
\text { Rápida }\end{array}$ \\
\hline $\begin{array}{c}\text { Custo de } \\
\text { implementação }\end{array}$ & Nenhum & Baixo & $\begin{array}{c}\text { Extremamente } \\
\text { Elevado }\end{array}$ \\
\hline
\end{tabular}

\section{CONCLUSÕES}

No presente artigo foram apresentadas as principais propostas para o amortecimento do fluxo do estator da máquina trifásica de dupla alimentação com conversor estático em seu circuito rotórico.

Estando a máquina operando com geração de potência reativa, os limites de estabilidade podem ser atingidos e o fluxo do estator passa a apresentar oscilações com frequência próxima à frequência da rede. De forma a eliminar estas oscilações, três diferentes estratégias foram apresentadas. A primeira consiste em ajustar os parâmetros dos controladores de corrente de tal forma que estas malhas de controle fiquem insensíveis a oscilações com frequência próxima à frequência do sistema elétrico. A segunda proposta visa corrigir o problema a partir das correntes rotóricas, através de um termo adicional inserido nas referências das correntes nos eixos direto e em quadratura. A terceira e última estratégia apresentada visa corrigir o problema diretamente no estator, através da inserção de um inversor adicional em série com o circuito do estator no lugar de seu ponto neutro, sendo este utilizado para variar as características dinâmicas da máquina.

Os melhores desempenhos no amortecimento das oscilações no fluxo ficaram por conta das duas últimas estratégias. Embora o amortecimento ativo através de variação dinâmica da planta possibilite estabilizar a máquina de forma extremamente rápida, principalmente após faltas no sistema elétrico, a estratégia de amortecimento back-ims apresenta a melhor razão custo/benefício.

\section{AGRADECIMENTOS}

Ao Institut für Regelungstechnik da TU Braunschweig por disponibilizar sua infra-estrutura para o desenvolvimento deste trabalho e ao DAAD pelo suporte financeiro.

\section{REFERÊNCIAS BIBLIOGRÁFICAS}

[1] C.R. Kelber, "Operação de turbinas hidráulicas em velocidade ajustável", Engenharia - Estudos Tecnológicos, ISSN 0101-5303, Vol. XXIII, No. 20, pp.5-13, 2000 (2002).

[2] C.R. Kelber, W. Schumacher, "Adjustable Speed Constant Frequency Energy Generation Technologies for Micro, Mini and Small Hydroelectric Power Plants", Engenharia - Estudos Tecnológicos, ISSN 0101-5303, Vol. XX, No. 23, pp.27-35, 2002 (2003).

[3] C.R. Kelber, W. Schumacher, "Adjustable Speed Constant Frequency Energy Generation with DoublyFed Induction Machines", VSSHy 2000 - European Conference Variable Speed in Small Hydro, Grenoble, Jan. 2000 .

[4] C.R. Kelber, W. Schumacher, "Control of Doubly-Fed Induction Machines as an Adjustable Speed Motor/Generator", VSSHy 2000 - European Conference Variable Speed in Small Hydro, Grenoble, Jan.2000.

[5] R. Richter, Elektrische Maschinen, Bd.IV - Die Induktionsmaschinen, Birkhäuser Verlag, 1954.

[6] W. Leonhard, Control of Electrical Drives, $2^{\text {nd }}$. Ed., Springer, 1996.

[7] A. Bellomi, J.M. Pacas, H. Späth , "Untersuchung der Stabilität der doppeltgespeisten Drehstrommaschine mit Hilfe eines linearisierten Modells", Archiv für Elektrotechnik, Nr.67, pp. 161-167, 1984.

[8] H. Stemmler, "Converter Controlled Fixed-Frequency Variable-Speed Motor / Generator", IPEC-Yokohama '95, pp. 170-176, 1995.

[9] A.M.Wlaczyna, W. Koczara, "Simulation Study of Dynamics of Current-Controlled Doubly-Fed Induction Machine", EPE'89, pp. 876-881, 1989.

[10] S. Wang, Y. Ding, "Stability Analysis of Field Oriented Doubly-Fed Induction Machine Drive Based on Computer Simulation", Electric Machines and Power Systems, 21:11-24, pp. 11-24, 1993.

[11] M. Heller, W. Schumacher, "Stability Analysis of Doubly-Fed Induction Machines in Stator Flux Reference Frame", EPE'97, pp. 2.707-2.710, 1997.

[12] J.M. Merino, "Effizienterer und flexiblerer Betrieb von Wasserkraftwerken mit Varspeed-Generatoren", $A B B$ Technik, No.3, pp. 33-38, 1996.

[13] C.R. Kelber, Aktive Dämpfung der doppelt-gespeisten Drehstrommaschine, Diss. TU-Braunschweig, 2000.

[14] Y. Kawabata, E.C. Ejiogu, T. Kawabata, "High Performance Drive System of Wound Rotor Induction Motor Using Two Current Controlled Inverters", PEMC 98, pp. 8.201-8.206, 1998.

[15] D. Lecocq, Ph. Lataire, "The Indirect-Controlled Double Fed Asynchronous Motor for Variable-Speed Drives", EPE'95, pp. 3.405-3.410, 1995.

[16] D. Ramuz, J.M. Kauffmann, A. Mirzzaian, S. Tnani, H. Godfroid, "Robustness of a Doubly Fed Machine Control Law to Load Variation", Electrimacs 99, pp. II.123-II.128, 1999.

[17] C.R. Kelber, W. Schumacher, "Active damping of flux oscillations in doubly-fed AC machines using dynamic 
variation of the system's structure", EPE2001, Graz, Austria, 27-29 Aug. 2001.

[18] E. Kiel, W. Schumacher, "VeCon: A High-Performance Single-Chip-Servocontroller", IPEC-Yokohama '95, pp. 1284-1289, 1995.

[19] Popenda, Z. Krzeminski, "Damping of Flux and Current Oscillations in Double Fed Asynchronous Machine", PEMC 96, pp. 3/614-3/618, 1996.

\section{DADOS BIOGRÁFICOS}

Christian R. Kelber, nascido em 24/02/1972 em Erlangen, é formado em Engenharia Elétrica nas ênfases de Sistemas de Potência e Eletrônica pela PUC-Rio (1995), mestre (1997) pela mesma instituição e doutor em Engenharia Elétrica (2000) pela Technische Universität Carolo-Wilhelmina zu Braunschweig, na Alemanha, tendo desenvolvido sua tese no Institut für Regelungstechnik sob a orientação do Prof.Dr.Ing. Walter Schumacher. Desde 2001 é professor adjunto da Universidade do Vale do Rio dos Sinos - UNISINOS. Suas áreas de interesse são: Controle Aplicado, Tecnologias para Automação Veicular, Veículos Autônomos e Controle de Máquinas Elétricas.

Walter Schumacher, nascido em 26/10/1952 em Hamburg, é Professor Doutor (Prof.Dr.-Ing.) e Diretor do Institut für Regelungstechnik da Technische Universität CaroloWilhelmina zu Braunschweig, na Alemanha. Doutor pelo "Instituto de Engenharia de Controle" do qual já foi pesquisador e desde 1993 é diretor, dá continuidade ao trabalho iniciado pelo Prof.Dr-Ing. Werner Leonhard. Na indústria alemã chefiou o Setor de Sistemas da empresa Institut für Angewandte Mikroelektronik GmbH (IAM), onde foram desenvolvidos, dentre outras soluções, processadores digitais de sinais para aplicações em controle de máquinas elétricas. É membro dos seguintes comitês:

- "Executive Committee and International Steering Committee of EPE"

- "International Steering Committee of PEMC"

- "International Steering Committee of Electrimacs"

Também é membro das seguintes instituições:

- VDI, VDE, IEEE e EPE Association

\section{APÊNDICE}

\section{Tabela A1: Dados da MTDA}

Dados do Gerais:

Potência nominal

Fator de potência

Frequência nominal (estator)

Número de par de pólos

Dados do Estator:

Tensão nominal

Corrente nominal

Resistência

Indutância

Fator de dispersão

Dados do Rotor:

Tensão nominal (com eixo travado)

Corrente nominal

Resistência

Indutância

Fator de dispersão

$\begin{array}{ll}\mathrm{P}_{\mathrm{S} 0} & =33 \mathrm{~kW} \\ \cos (\varphi) & =0.84 \\ \mathrm{f}_{0} & =50 \mathrm{~Hz} \\ \mathrm{p}_{\mathrm{p}} & =2 \\ \mathrm{U}_{\mathrm{S} 0} & =400 \mathrm{~V} \\ \mathrm{I}_{\mathrm{S} 0} & =61 \mathrm{~A} \\ \mathrm{R}_{\mathrm{S}} & =0.072 \Omega \\ \mathrm{L}_{\mathrm{S}} & =0.0279 \mathrm{H} \\ \sigma_{\mathrm{S}} & =0.0232 \\ \mathrm{U}_{\mathrm{r} 0} & =800 \mathrm{~V} \\ \mathrm{I}_{\mathrm{r} 0} & =30 \mathrm{~A} \\ \mathrm{R}_{\mathrm{r}} & =0.346 \Omega \\ \mathrm{L}_{\mathrm{r}} & =0.1176 \mathrm{H} \\ \sigma_{\mathrm{r}} & =0.0352\end{array}$

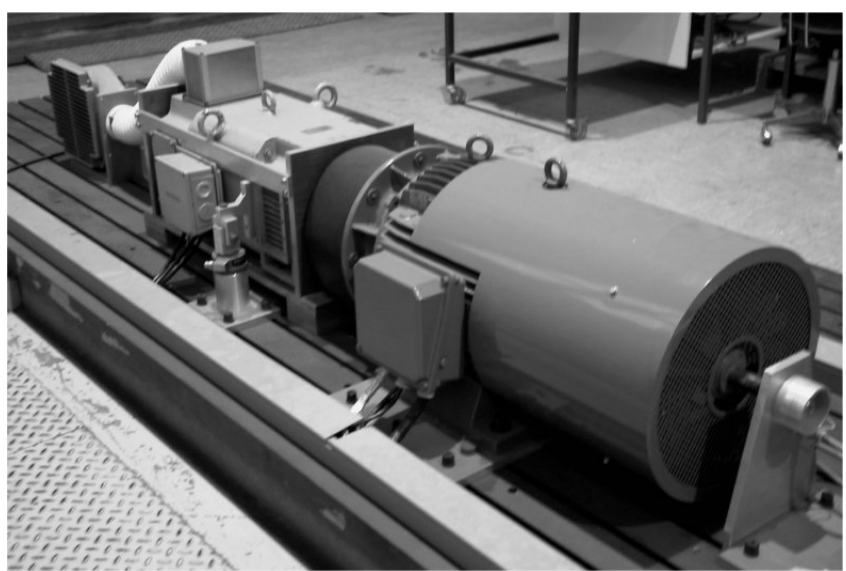

Figura A1: MTDA acionada por uma máquina CC 